\title{
Person Verification Using Intra-Palm Propagation Signals with A New Phase Spectrum
}

\author{
$1^{\text {st }}$ Kohei Fujita \\ Graduate School of Sustainability Sciences \\ Tottori University \\ Tottori, Japan \\ m19j4042b@edu.tottori-u.ac.jp
}

\author{
$2^{\text {nd }}$ Yuya Ishimoto \\ Graduate School of Sustainability Sciences \\ Tottori University \\ Tottori, Japan \\ m18j4004k@edu.tottori-u.ac.jp
}

\author{
$3^{\text {rd }}$ Isao Nakanishi \\ Faculty of Engineering \\ Tottori University \\ Tottori, Japan \\ nakanishi@tottori-u.ac.jp
}

\begin{abstract}
In recent years, biometrics such as fingerprints and iris scans has been used in authentication. However, conventional biometrics is vulnerable to identity theft, especially in user management systems. As a new biometric without this vulnerability, we focused on intra-palm propagation signals, which are not exposed on a body surface. An intra-palm propagation signal is a signal that propagates between two pairs of electrodes, which are on the transmitter and receiver sides on a palm. When current passes between the electrodes on the transmitter side, the leakage electric field is generated. On the receiver side, this electric field is detected as a voltage change and regarded as an intra-palm propagation signal. In this paper, a new feature based on a phase spectrum of an intra-palm propagation signal is proposed, and its performance for verifying individuals is examined by the evaluation.
\end{abstract}

Index Terms-biometrics, intra-palm propagation signal, phase spectrum, verification performance

\section{INTRODUCTION}

At present, our daily lives cannot be imagined without the use of digital information devices such as smartphones, PCs, and tablets. In such an information society, leakage of personal information data can cause severe damage, so strengthening security is becoming very important. Personal authentication that verifies whether applicants who want to use a system are genuine (registered) users is one of the possible ways to strengthen the security of smartphones, PCs, and tablets. In the user authentication procedures, passwords, or IC cards are used, but they have the risk of being stolen, forgotten, or lost.

Therefore, biometric-based authentication has been recently built in smartphones and bank ATMs [1]. This kind of authentication solves the above-mentioned problem because biometrics can never be forgotten or lost; thus, user burden is decreased. In typical biometric-based authentication, users are identified by their fingerprints, faces, irises, voice prints, and signatures, which all denote personal characteristics. However, conventional biometric-based authentication has certain drawbacks. One of them is that biometric information exposed on the user body surface can be stolen by others and used for false identification. Also, conventional biometric-based authentication assumes one-time-only authentication, which cannot prevent the spoofing attack by imposters.

With the aim to overcome these drawbacks, we proposed to use the intra-palm propagation signals as biometrics [2]-

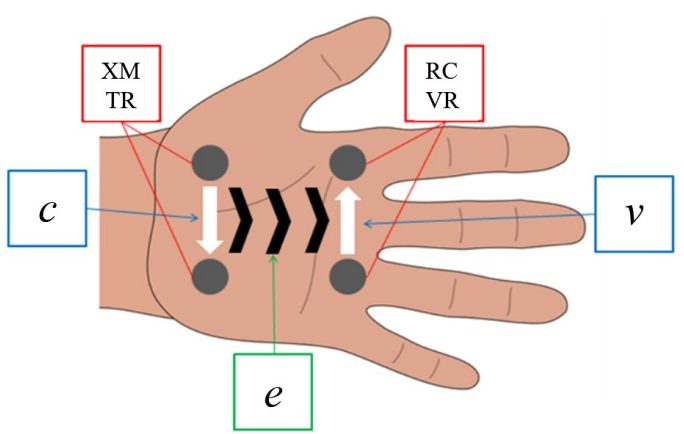

Fig. 1. Intra-palm propagation signal.

[5]. The intra-palm propagation signal is not exposed on the human body surface. In addition, if a mobile terminal, e.g., a smartphone, is equipped with a measuring device for intra-palm propagation signals, its users can be continuously authenticated while holding the terminal. To use the intra-palm propagation signal as biometrics is novel; therefore, there is no comparable biometrics.

We have used a composite sine-wave having multiple frequencies, which propagates on a palm [2]-[5]. From the propagated signal, amplitude and phase spectra are extracted using the fast Fourier transform (FFT) and then are used as individual features. The extracted features are used to verify user identity. However, the verification using the phase spectrum has lower performance compared with that using the amplitude spectrum. In particular, the equal error rate (EER) is $51 \%$ using the Euclidian distance matching, and $33 \%$ using the support vector machine [4]. In this paper, we propose a new phase spectrum to improve verification performance.

\section{IntRa-PALM Propagation SignAL}

Figure 1 shows a mechanism for detecting an intra-palm propagation signal. Two pairs of electrodes are installed on a palm, and current $(c)$ is passed through the pair of electrodes at the transmitter (XMTR) side. At that time, the leakage electric field $(e)$ is generated around the electrodes, and it propagates on the palm. The generated electric field is detected as a voltage change $(v)$ on the receiver (RCVR) side, which is called the intra-palm propagation signal [2]. 


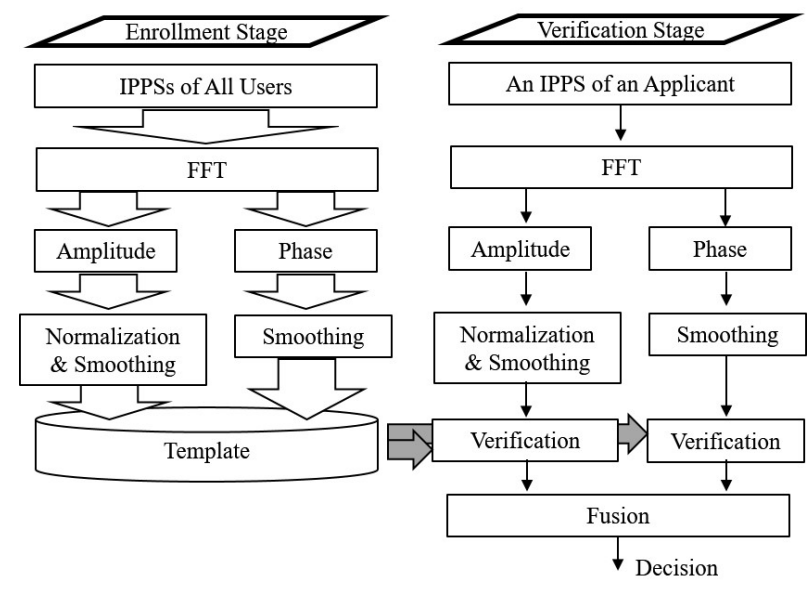

Fig. 2. User verification procedure.

According to the guideline provided by the International Commission on Non-Ionizing Radiation Protection (ICNIRP) [6], an allowable current that can safely pass through the human body without hurting it is up to $20 \mathrm{~mA}$ in the frequency range from $100 \mathrm{kHz}$ to $110 \mathrm{MHz}$. Therefore, in measuring the intra-palm propagation signals, the current has to be kept below $20 \mathrm{~mA}$.

\section{Person Verification Using Phase Spectrum}

In Refs. [3]-[5], the intra-palm propagation signal has been analyzed using a discrete Fourier transform (DFT), while the FFT has been used in practice, and amplitude and phase spectra have been used as individual features.

\section{A. Verification System}

In this study, we study user verification, where users are verified as being genuine or not. The procedures are illustrated in Fig. 2. In the enrolment stage, the intra-palm propagation signals (IPPSs) are measured from all the users, and then their amplitude and phase spectra are obtained by the FFT as individual features. A template is a comparative target in matching, which is used in the verification stage and generated by ensemble-averaging several intra-palm propagation signals of each subject. In the verification stage, an applicant who wants to access the system specifies one of the enrolled users, then his/her intra-palm propagation signal is measured, and its amplitude and phase spectra are obtained. The two obtained spectra are used to judge whether the applicant is genuine or not. The judgment is based on the Euclidian distance matching by comparing the obtained value with that in the template relevant to the user specified by the applicant. The results obtained from the amplitude and phase spectra are integrated by a fusion rule, such as score level fusion or decision level fusion [7]. Finally, a fused result is compared with a threshold; if the result is smaller than the threshold, the applicant is regarded as a genuine user; otherwise, the applicant is regarded as an imposter. The threshold is empirically determined.

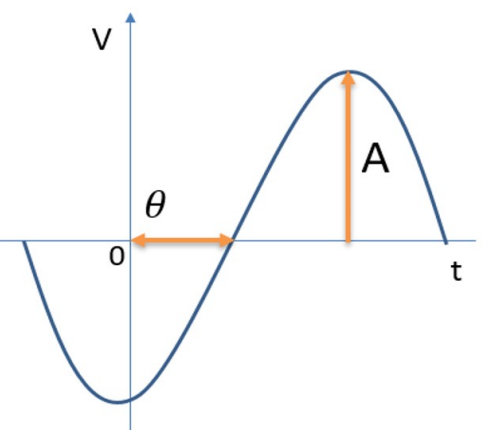

Fig. 3. Definition of a sine wave.

\section{B. Phase Spectrum of Intra-Palm Propagation Signal}

Amplitude and phase spectra are obtained by DFT (FFT). The DFT is defined as:

$$
\begin{aligned}
X_{k} & =\sum_{n=0}^{N-1} x_{n} \exp ^{-j \frac{2 \pi n k}{N}} \\
& =\sum_{n=0}^{N-1} x_{n}\left\{\cos \left(\frac{2 \pi n k}{N}\right)-j \sin \left(\frac{2 \pi n k}{N}\right)\right\} \\
& =a(k)+j b(k),
\end{aligned}
$$

where $x(n)(n=0,1, \ldots, N-1)$ denotes sampled data of an input signal, and $n$ and $k$ are a time index and a frequency index, respectively; $a$ is the real part, $b$ is the imaginary part, and $j$ is the imaginary unit. The amplitude and phase spectra are respectively defined as follows:

$$
\begin{gathered}
A(k)=\sqrt{a(k)^{2}+j b(k)^{2}}, \\
\theta(k)=\tan ^{-1}\left(\frac{b(k)}{a(k)}\right) .
\end{gathered}
$$

In general, for complex numbers, the real part is the base. In the DFT, the cosine wave is a reference, as shown in Eq. (1). When a sine wave having the phase of zero is analyzed, the phase spectrum is $(-\pi / 2)$ at all the frequencies.

\section{Signal Truncating Influence on Phase}

A sine wave with an amplitude $A$ and a phase $\theta$ is defined as $A \sin (2 \pi f t+\theta)$, as shown in Fig. 3. We have used a composite of sine waves with the same amplitudes, phases of zero, and

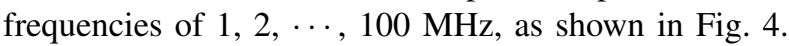

Propagation of the sin signal on a palm can cause a phase delay or lead at every frequency, which can be seen in the phase spectrum. If the phase delay or lead differs from person to person, so it can be used as a feature for distinguishing individuals. However, in general, a propagation signal and its propagated signal are not synchronized since synchronization of two signals requires double-measuring devices and accurate detection of starting points of both signals, which is not feasible in practical applications. Therefore, as shown in Fig. 5 , even when the same propagation signal is analyzed, if the timings for truncating the sampled data for the DFT analysis 


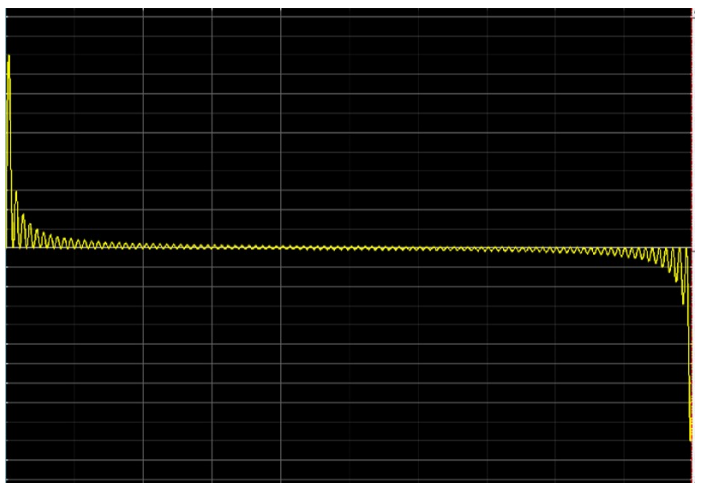

Fig. 4. The composite sine wave propagating on a palm.

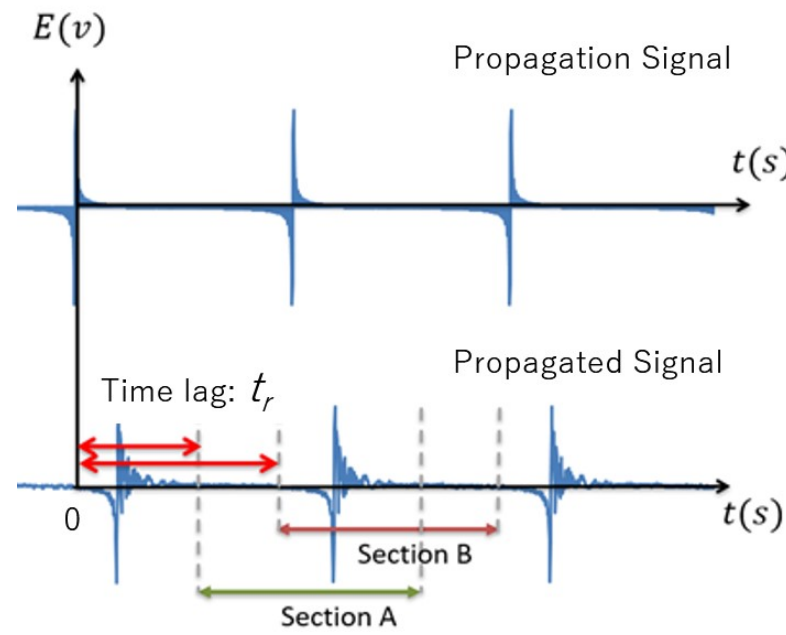

Fig. 5. Truncation of sampled data from the propagated signal.

from the signal are different (e.g., Sections A and B), it causes a different time-delay or lead, that is, a different phase spectrum. Let the time lag $t_{r}$ and a time difference caused by propagation on a palm $t_{k}$, then the phase at each frequency $k f$ is defined as:

$$
\theta(k)=2 \pi k f\left(t_{k}+t_{r}\right) .
$$

\section{Phase Indefiniteness}

A trigonometric function has a property of $\sin (x)=\sin (x \pm$ $2 \pi m$ ), where $m$ represents the nature number. Therefore, the phase over $\pm 2 \pi$ cannot be distinguished from that under $\pm 2 \pi$. In other words, the phase spectrum has indefiniteness of $2 \pi$, which represents a problem in the phase spectrum extraction As given in Eq. (3), the phase is defined as an inverse tangent function; therefore, its value can be calculated only in the range from $\pi / 2$ to $-\pi / 2$. However, if a phase spectrum has a characteristic that frequency elements increase in proportion to the frequency values, it is necessary to obtain the phase spectrum over $\pm \pi / 2$.

Accordingly, the unwrapping process is necessary to obtain a phase spectrum over $\pm \pi / 2$. The phase spectra before and

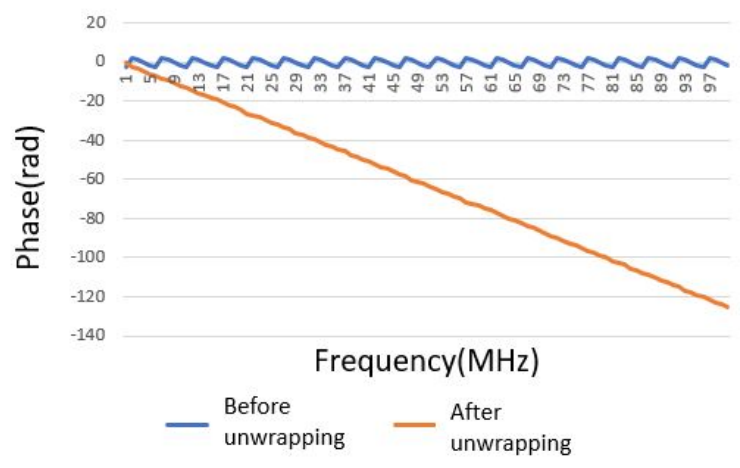

Fig. 6. Phase spectra before and after unwrapping.

after the unwrapping process are presented in Fig. 6 . The phase spectrum before unwrapping can be obtained only in the range from $\pi / 2$ to $-\pi / 2$ because of its indefiniteness. On the other hand, the proportional phase spectrum to frequencies can be obtained over $\pm \pi / 2$ by introducing unwrapping.

\section{Second-Order Difference Phase Spectrum}

According to the phase in Eq. (4), the time lag $t_{r}$ caused by the signal truncation for the DFT is constant in the time domain regardless of frequency, but in the frequency domain, its phase component $2 \pi k f t_{r}$ is proportional to the frequency after the unwrapping, as shown in Fig. 6. This phenomenon is known as a linear-phase phenomenon in signal processing. On the other hand, a phase component $2 \pi k f t_{k}$ generated by signal propagation on a palm can have various values at each frequency. In this work, we aim to remove the constant time lag, which is presented as a slope in the phase spectrum, and extract only the variations at frequencies, which can be used as individual features. Thus, the second-order differential of the phase spectrum is introduced, which is defined as:

$$
P(k)=\frac{d^{2}}{d \omega^{2}} \theta .
$$

However, the actual phase spectrum is discrete, and thus cannot be differentiated. Therefore, we calculate the secondorder difference. The first-order difference of the phase spectra is given by:

$$
\begin{aligned}
\theta_{d}(k) & =\theta(k+1)-\theta(k) \\
& =2 \pi(k+1) f t_{k+1}-2 \pi k f t_{k}+2 \pi f t_{r} .
\end{aligned}
$$

And, its second-order difference is expressed as:

$$
\begin{aligned}
\theta_{d d}(k)= & \theta_{d}(k+1)-\theta_{d}(k) \\
= & \theta(k+2)-\theta(k+1)-(\theta(k+1)-\theta(k)) \\
= & \theta(k+2)-2 \theta(k+1)-\theta_{d}(k) \\
= & 2 \pi(k+2) f t_{k+2}-4 \pi(k+1) f t_{k+1} \\
& +2 \pi k f t_{k} .
\end{aligned}
$$

According to Eq. (7), the time lag $t_{r}$ generated by the signal truncation is removed, and time differences, $t_{k+2}, t_{k+1}$, and $t_{k}$, are extracted as individual features. The examples in which 


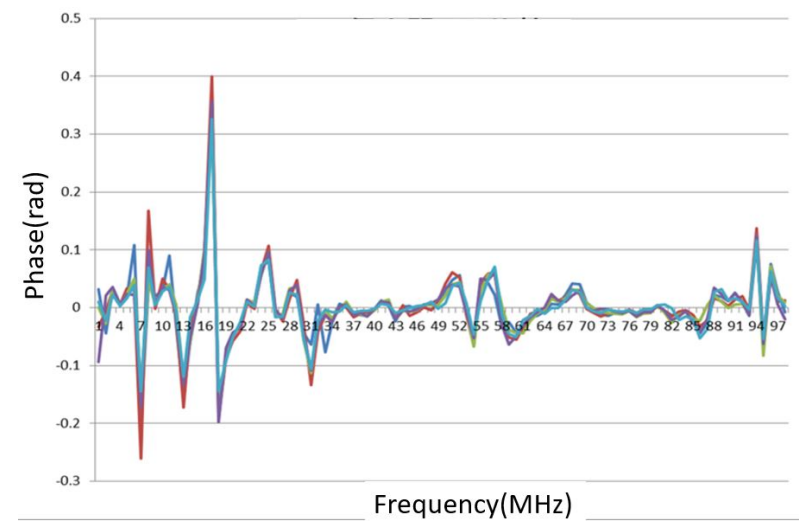

Fig. 7. Examples of the second-order difference in phase spectra.

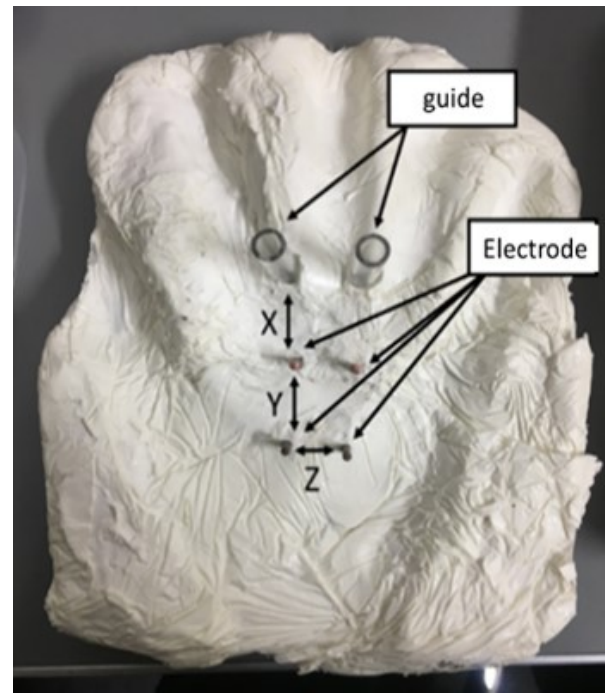

Fig. 8. Dedicated measuring device [3].

the second-order difference is applied to the phase spectra of five experimental subjects are presented in Fig. 7. As displayed in Fig. 7, there is no bias or slant in the phase spectra.

\section{Evaluation of Proposed Phase Spectrum}

The proposed phase spectrum was evaluated by verifying individuals using the intra-palm propagation signals, which can be found in Ref. [3]. The measurement method and conditions are described in the following.

\section{A. Intra-Palm Propagation Signals Database}

In the measurement of intra-palm propagation signals, putting an electrode on a palm may cause fluctuations in phase spectrum characteristics even for the same user. Therefore, a dedicated measurement device with a guide for fixing the position of electrodes on a palm was prepared, and it is shown in Fig. 8. In addition, the size of palms was different, which also resulted in fluctuation in phase spectrum characteristics. Therefore, three dedicated measuring devices with different sizes, $\mathrm{S}, \mathrm{M}$, and $\mathrm{L}$, were prepared. Considering the average

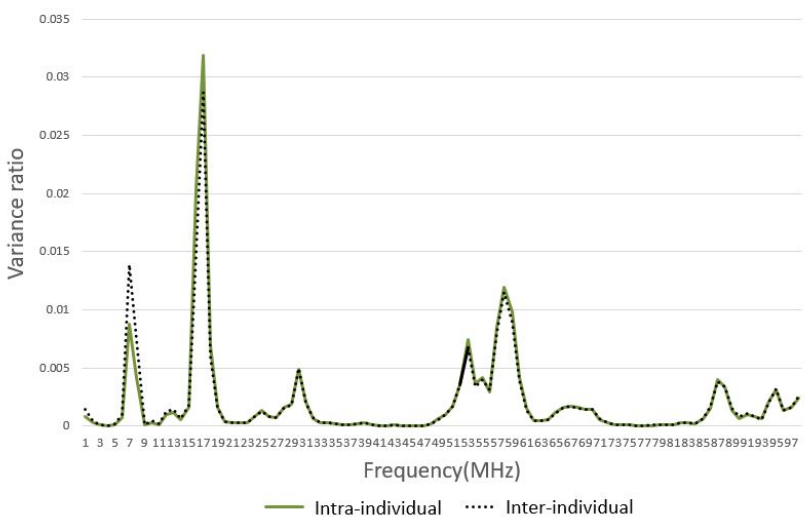

Fig. 9. Inter- and intra-individual variations in the second-order difference phase spectrum.

palm size of Japanese, electrode positions corresponding to the dedicated measuring device of size $\mathrm{M}$ were used, and those of sizes $\mathrm{S}$ and $\mathrm{L}$ were determined based on the reference to that of size M. Measurement of the intra-palm propagation signals was performed on 15 experimental subjects in about 10 days up to two times a day, keeping the time interval between two successive measurements be at least 4 hours. At the end of the measurement process, 20 measured datasets per each subject were collected. The signal propagated from the transmitter was a composite sine wave shown in Fig. 4.

\section{B. Frequency Selection Using Dispersion Ratio}

In general, biological information such as an intra-palm propagation signal varies for different but even for the same individuals, and these variations are called the intra-individual variation and the inter-individual variation, respectively. The biological information that shows a small intra-individual variation and a large inter-individual variation is suitable for person (e.g., user) verification. Thus, we evaluated inter- and intra-individual variations in the second-order difference phase spectrum, and the results are shown in Fig. 9. Although the differences between the intra- and inter-individual variations were not large, there were some cases where inter-individual variations were larger than intra-individual variations; for instance, at $1 \mathrm{MHz}, 8 \mathrm{MHz}$, and $10 \mathrm{MHz}$.

Thus, we calculated the variance ratio by dividing an interindividual variation by an intra-individual variation every frequency. The variance ratios at all frequencies are shown in Fig. 10. The ratio of lager than one indicated that interindividual variations were larger than the corresponding intraindividual variations, and those frequencies were considered as suitable for verification of individuals. In the lower frequency band, there were frequencies suitable to be used in the user verification. The reason for such results is not clear, but it could be due to the fact that in the measurement, we used a digital oscilloscope as a receiver having the sampling frequency of $200 \mathrm{MHz}$; therefore, higher frequency spectral elements might not be detected accurately. In our future work, 


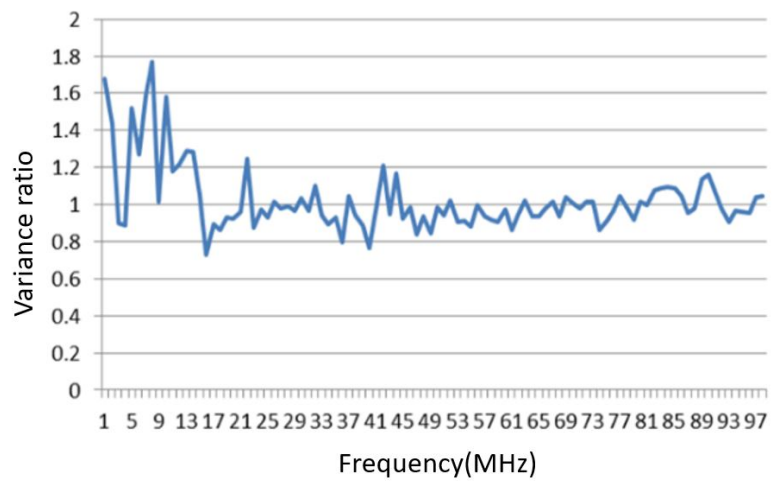

Fig. 10. Variance ratios.

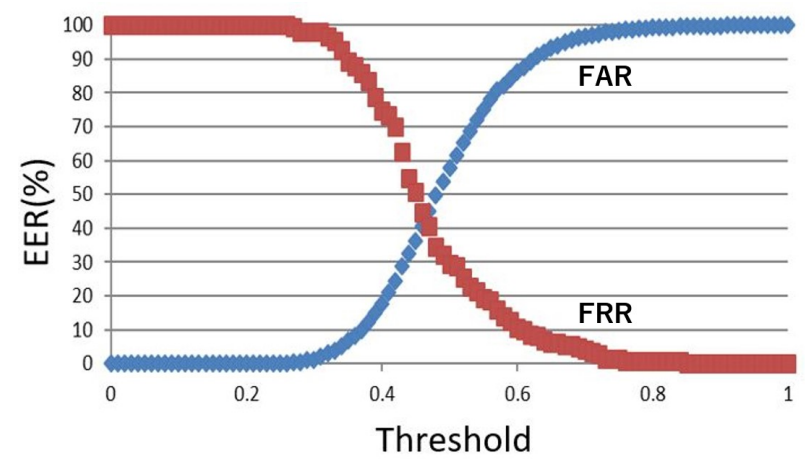

Fig. 11. Error rate curves.

we will investigate the reason why effective frequencies exist in a lower frequency band.

\section{Verification by Euclidean Distance Matching Method}

The verification using the proposed second-order difference phase spectrum was evaluated by the Euclidean distance matching method. First, all 98 elements in the second-order difference phase spectrum were used as individual features in the verification process. Ten genuine phase spectra of each subject were averaged to make a template, and the remaining ten datasets were used in testing.

In an authentication system, the false acceptance rate (FAR) that is the rate of accepting imposters and the false rejection rate (FRR) that is the rate of rejecting genuine users are used and there is a trade-off between these rates. Therefore, for performance evaluation, we use the equal error rate (EER), where FAR $=$ FRR. Figure 11 shows the FRR and FAR curves, and the EER was $42.7 \%$. By comparing our EER with the EER of $51 \%$ reported in Ref. [4], it can be concluded that the proposed phase spectrum is more effective than the one reported in Ref. [4].

Next, we examined the EER changing trend by increasing the number of frequencies one by one using the order of effective frequencies presented in Section V-B. Figure 12 shows the change in the EER with the number of frequencies. According to the results, the EER has the best value of 35.1

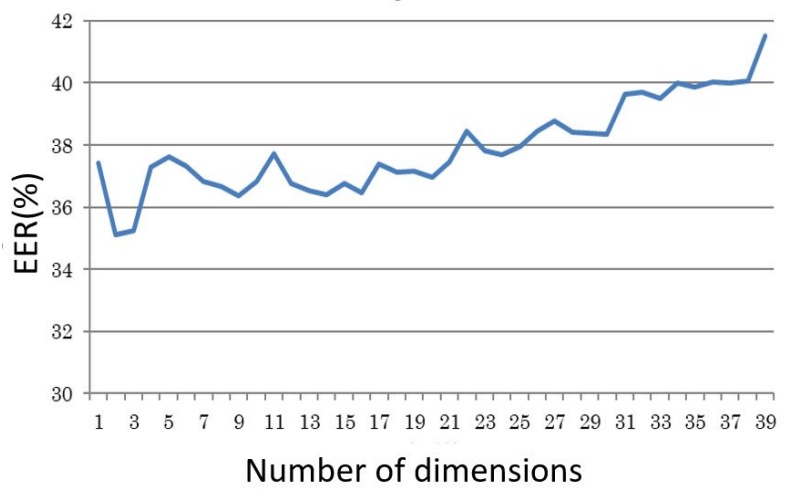

Fig. 12. The EER changing trend with frequencies.

$\%$ when the number of frequencies was 2 , i.e., when the $1^{\text {st }}$ and $8^{\text {th }}$ frequencies were used in the verification process. The EERs does not decrease even when the number of frequencies was increased. Therefore, it was found that the optimal number of feature dimensions was 2 or 3 .

\section{CONCLUSIONS}

In order to improve the verification performance using the intra-palm propagation signals, we propose the second-order difference phase spectrum and frequency selection based on the change in the intra- and inter-individual variations. The verification performance of the proposed feature is verified by the Euclidean distance matching method. The results showed that the EER of approximately $35 \%$ was obtained, which makes the proposed method still inconvenient to be used in practical applications. In our future work, we will evaluate other extracting methods of phase spectra to improve the verification performance further. In addition, we will fuse the result obtained by using the proposed phase spectrum with that obtained by using the amplitude spectrum. Also, we will introduce some intelligent rule for fusing the results.

\section{REFERENCES}

[1] J. Wayman, A. Jain, D. Maltoni, and D. Maio, Biometric Systems, Springer, 2004

[2] T. Inada, Y. Sodani, and I. Nakanishi, "Intra-Palm Propagation Signals as Suitable Biometrics for Successive Authentication," Journal of Computer Technology and Application, Vol. 7, No. 2, pp. 65-72, Feb. 2016

[3] I. Nakanishi, I. Ogushi, R. Nishi, and T. Murakami, "Effect of Propagation Signal and Path on Verification Performance Using Intra-Body Propagation Signals," Proc. of 2017 International Conference on Biometrics Engineering and Application (ICBEA2017), pp. 80-84, Apr. 2017

[4] M. Tokuda, and I. Nakanishi, "Personal Identification Using Phase Spectrum of Intra-palm Propagation Signal (in Japanese)," Proc. of the 2017 IEICE Society Conference, p. 153, Sep. 2017

[5] Y. Ishimoto, and I. Nakanishi, "A study on Individual Authentication Using Intra-Palm Propagation Signals - Fusion of Amplitude and Phase Spectral Features -," Proc. of 2018 International Symposium on Multimedia and Communication Technology (ISMAC2018), pp. 53-56, Aug. 2018

[6] International Commission on Non-Ionizing Radiation Protection (ICNIRP), "Guidelines for limiting exposure to time-varying electric magnetic and electromagnetic fields," Oberschleissheim, Germany, pp. 513514, 1997

[7] A. Ross, K. Nandakumar, and A. Jain, Handbook of Multibiometrics, Springer US, 2006 